\title{
Balance de una producción combinada: madera de nogal y avellana
}

\section{Balance of a combined production: walnut wood and hazelnut}

\author{
Vilanova,A. ${ }^{*}$, Garcia, D. ${ }^{1}$, Abelló, L. ${ }^{2}$, Rovira, M. ${ }^{2}$, Aletà, N. ${ }^{1}$ \\ ${ }^{1}$ IRTA-Torre Marimon, 08140 Caldes de Montbui (Barcelona) \\ ${ }^{2}$ IRTA-Mas de Bover, 43120 Constantí (Tarragona) \\ *Autor para correspondencia: antonivilanova@gmail.com
}

\section{Resumen}

Las plantaciones mixtas se presentan como una alternativa dirigida a mejorar el balance productivo-económicoambiental a través de una óptima compartimentación del hábitat con combinaciones de especies complementarias. Evaluamos tres plantaciones de nogal para madera con acompañamiento de avellano en Caldes de Montbui (Barcelona, 8 años de edad), Prades (Tarragona, 9 años) y Ontinar de Salz (Huesca, 10 años). Se comparan el sistema mono-específico clásico de nogal, a marco $6 \times 6 \mathrm{~m}$ y 7x7 m, frente a un sistema con igual marco pero con acompañamiento de dos matas de avellano a ambos lados, a una distancia entre $0.75-1.50 \mathrm{~m}$. Establecimos registros de crecimiento primario y secundario, cantidad y tipo de ramificación y conformación del fuste del nogal hasta una altura de $3.0 \pm 0.5 \mathrm{~m}$. En el avellano se midió el diámetro de copa y se estimó la producción de fruto. El tratamiento de acompañamiento generó fustes de nogal más rectos y menos elípticos, con menor número y diámetro de ramas y nudos. La conclusión indica que el control de la distancia entre ambas especies y la elección de un material vigoroso de avellano, de crecimiento acompasado con el nogal, puede favorecer una producción de madera de mayor calidad y una disminución del esfuerzo de poda. Además, con la producción de avellana, se introduce un retorno económico anual respecto a plantaciones monoespecíficas, siempre y cuando se pueda compensar la superior demanda hídrica del sistema.

\section{Summary}

Mixed plantations are presented as an alternative aimed at improving the productive-economic-environmental balance through an optimal compartmentalization of the habitat with combinations of complementary species. We evaluated three walnut plantations for wood with hazelnut accompaniment in Caldes de Montbui (Barcelona, 8 years old), Prades (Tarragona, 9 years old) and Ontinar de Salz (Huesca, 10 years old). The classic mono-specific walnut system is compared to a $6 \times 6 \mathrm{~m}$ and $7 \times 7 \mathrm{~m}$ frame, compared to a system with the same frame but with the accompaniment of two hazel bushes on both sides, at a distance between $0.75-1.50 \mathrm{~m}$. We established records of primary and secondary growth, amount and type of branching and conformation of the walnut tree to a height of $3.0 \pm 0.5 \mathrm{~m}$. In the hazelnut, the crown diameter was measured and fruit production was estimated. The accompanying treatment resulted in more straight and less elliptical walnut stems, with fewer branches and knots. The conclusion indicates that the control of the distance between both species and the choice of a vigorous material of hazelnut, of growth measured with the walnut, can favor a production of wood of higher quality and a reduction of the effort of pruning. Furthermore, with the production of hazelnut, an annual economic return is introduced with respect to monospecific plantations, provided that the higher water demand of the system can be compensated.

Palabras clave: agroforestal, Corylus, Juglans, plantaciones mixtas.

Keywords: agroforestry, Corylus, Juglans, mixed plantations. 


\section{Introducción}

Las plantaciones para producción de madera de frondosas en Europa, particularmente de cerezo y de nogal, se están desarrollando ininterrumpidamente desde hace más de 20 años. En España algunos autores estiman entre 8000 y 10000 las hectáreas destinadas a estos cultivos (Aletà y Vilanova, 2014). Sin embargo, aunque se haya demostrado que las plantaciones mixtas y/o sistemas agroforestales parecen mejorar el balance productivo y ambiental (Becquey, 2006; Chifflot et al., 2006; Piotto, 2008), la gran mayoría de estas plantaciones siguen siendo monoespecíficas. En muchas de estas plantaciones monoespecíficas se han cuantificado bajos porcentajes de madera de calidad en observaciones realizadas en pie (Nosenzo et al., 2011, Vilanova et al., 2011). Entre los defectos observados sobre el fuste se citan un número y diámetro elevado de nudos, presencia frecuente de curvaturas y quemaduras solares sobre la corteza y cambium. Además, en este tipo de plantaciones la producción se centra únicamente en la madera, lo que implica un retorno económico lejano (actualmente se barajan turnos de entre 25-50 años) que se convierte en el principal freno de muchas iniciativas de inversión.

En este trabajo se proponen los sistemas agroforestales como parte de la solución. La fórmula se basa en introducir una especie frutal ampliamente conocida como es el avellano (Corylus avellana L.), dentro del sistema clásico de producción de madera de nogal, con la intención de mejorar la calidad de la madera, el retorno económico de la inversión y aumentar la biodiversidad. Los objetivos de este ensayo se basan en:

a) Detectar y cuantificar la mejora que sobre el fuste del nogal puede aportar el acompañamiento de avellano.

b) Calcular el balance del sistema en términos de producción de avellana, consumo hídrico y esfuerzo de poda.

\section{Materiales y métodos}

\subsection{Parcelas y material vegetal}

Los ensayos se realizaron en tres estaciones diferentes denominadas: "CALDES", "PRADES", y "ONTINAR". Las características de las estaciones y de los ensayos se resumen en Tab. 1 y Tab. 2. Todos los ensayos, ubicados en terrenos agrícolas, se instalaron con soporte hídrico, de tipo localizado en los dos primeros y a manta en ONTINAR. El ensayo de CALDES se asienta en una finca del IRTA, el de PRADES en finca privada, y en ambos casos las plantaciones fueron diseñadas inicialmente como ensayo. La explotación de ONTINAR es privada y se planteó con fines exclusivamente productivos. La especie evaluada para producción de madera en todos los casos es el nogal y la acompañante el avellano. De tal manera que los tratamientos considerados fueron "nogal acompañado con avellano" (CON) y "nogal sin acompañamiento" (SIN). Los plantones de nogal fueron materiales de tipo $1+0$ de entre 40 y $60 \mathrm{~cm}$. Los de avellano fueron retoños auto-enraizados de 
un año, de entre 30 y $50 \mathrm{~cm}$, procedentes de pies madre del IRTA, formando una mezcla de diferentes variedades. En el caso de ONTINAR el material vegetal provino de viveros comerciales. En relación al riego aportado, en CALDES y PRADES se situaron dos emisores sobre el nogal y en presencia de acompañamiento, uno más sobre cada avellano. El riego se programó en base a las condiciones climáticas semanales ocupando un periodo aproximado de 4 meses al año, de mayo a agosto. Las dosis aplicadas fueron proporcionales al incremento anual de la superficie de copa del nogal y el avellano. En ONTINAR, no se tienen registros evolutivos del riego, pero podrían situarse por encima de los $6000 \mathrm{~m}^{3} \mathrm{ha}^{-1} \mathrm{año}^{-1}$.

Tabla 1. Características de la estación.

\begin{tabular}{|l|c|c|c|c|c|}
\hline \multirow{2}{*}{ Nombre } & \multicolumn{2}{|c|}{ Localización } & \multicolumn{2}{c|}{ Climatología } \\
\cline { 2 - 6 } & UTM & $\begin{array}{c}\text { Altura } \\
(\mathrm{msnm})\end{array}$ & $\begin{array}{c}\text { Tm anual } \\
\left({ }^{\circ} \mathrm{C}\right)\end{array}$ & $\begin{array}{c}\text { P anual } \\
(\mathrm{mm})\end{array}$ & Observaciones \\
\hline $\begin{array}{l}\text { CALDES } \\
\text { (Barcelona) }\end{array}$ & $\begin{array}{c}31 \mathrm{~N}: \\
431204-4607394\end{array}$ & 163 & 14.0 & 644 & $\begin{array}{c}\text { Clima mediterráneo } \\
\text { a 20 km de la costa }\end{array}$ \\
\hline $\begin{array}{l}\text { PRADES } \\
\text { (Tarragona) }\end{array}$ & $\begin{array}{c}331 \mathrm{~N}: \\
977-4576301\end{array}$ & 980 & 9.3 & 673 & $\begin{array}{c}\text { Veranos suaves } \\
\text { e inviernos fríos }\end{array}$ \\
\hline $\begin{array}{l}\text { ONTINAR } \\
\text { (Zaragoza) }\end{array}$ & $\begin{array}{c}30 \mathrm{~N}: \\
686797-4645418\end{array}$ & 311 & 14.0 & 311 & Continental seco \\
\hline
\end{tabular}

Tabla 2. Características de las parcelas de ensayo

\begin{tabular}{|c|c|c|c|c|c|}
\hline Nombre & $\begin{array}{c}\text { Año } \\
\text { plantación }\end{array}$ & $\begin{array}{l}\text { Superficie } \\
\text { (ha) }\end{array}$ & Especies & $\begin{array}{c}\text { Distancias } \\
\text { plantación }(\mathrm{m})\end{array}$ & Diseño \\
\hline $\begin{array}{l}\text { CALDES } \\
\text { (Barcelona) }\end{array}$ & 2008 & 0.7 & $\begin{array}{c}\text { Nogal: } \\
\text { Juglans } x \text { intermedia }{ }^{1} \\
\text { con y sin Avellano }{ }^{3}\end{array}$ & $\begin{array}{l}6 \times 6 \text { nogal } \\
\text { Avellano a } 0.75 \mathrm{~m} \text { del } \\
\text { nogal a cada lado }\end{array}$ & $\begin{array}{l}8 \text { árboles por } \\
\text { tratamiento } \\
\text { y } 3 \text { repeticiones }\end{array}$ \\
\hline $\begin{array}{l}\text { PRADES } \\
\text { (Tarragona) }\end{array}$ & 2007 & 0.4 & $\begin{array}{c}\text { Nogal: } \\
\text { Juglans regia }{ }^{2} \\
\text { con y sin Avellano }\end{array}$ & $\begin{array}{c}6 \times 6 \text { nogal } \\
\text { Avellano a } 1.5 \mathrm{~m} \text { del } \\
\text { nogal a cada lado }\end{array}$ & $\begin{array}{l}18 \text { árboles por } \\
\text { tratamiento } \\
\text { y } 3 \text { repeticiones }\end{array}$ \\
\hline $\begin{array}{l}\text { ONTINAR } \\
\text { (Zaragoza) }\end{array}$ & 2004 & 4.0 & $\begin{array}{c}\text { Nogal: } \\
\text { Juglans xintermedia } \\
\text { con y sin Avellano }\end{array}$ & $\begin{array}{l}7 \times 7 \text { nogal } \\
\text { Avellano a } 1.5 \mathrm{~m} \text { del } \\
\text { nogal a cada lado }\end{array}$ & $\begin{array}{c}\text { Muestreo } \\
\text { estratificado a } \\
\text { nivel de parcela. } \\
22 \text { con avellano } \\
\text { y } 21 \text { sin. }\end{array}$ \\
\hline
\end{tabular}

${ }^{1}$ Progenie comercial identificada como 'Ng23xRa'.

${ }^{2}$ Progenies de $J$. regia seleccionadas por el IRTA.

${ }^{3}$ Mezcla de variedades de avellano (Corylus avellana L.) identificadas en CALDES y PRADES. En ONTINAR se utilizaron dos variedades catalanas de uso comercial 'Negret' y 'Pauetet'.

\subsection{Toma de datos}

La toma de datos en CALDES y PRADES siguió un itinerario anual de mediciones en el que se registraron sobre el nogal el diámetro normal en cruz, la altura 
total, la altura de fuste sin ramas y el diámetro de copa en cruz respecto a la línea de plantación. En el avellano se midieron la altura y los diámetros de copa. La rectitud del nogal se registró sobre los primeros $2.5 \mathrm{~m}$ de fuste siguiendo la valoración establecida por Vilanova et al., (2011) en la que se define el fuste como: fuste curvado (comparable a una curvatura de flecha superior a $6 \mathrm{~cm} \mathrm{~m}^{-1}$ ), fuste ligeramen-

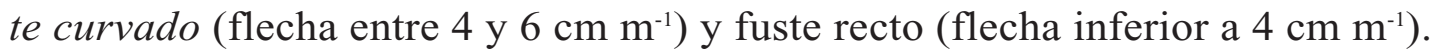

La elipticidad ${ }^{[1]}$ del fuste de nogal se evaluó a $1.30 \mathrm{~m}$ de altura en todos los ensayos y en CALDES además a $2.50 \mathrm{~m}$.

$$
E=\left(\frac{D-d}{D}\right) \times 100
$$

Siendo E la elipticidad en unidades porcentuales (con valores crecientes a mayor elipticidad), D el diámetro mayor registrado y d el diámetro menor, procedentes de las medidas de diámetro en cruz.

Sobre los 3 primeros metros de fuste, tanto en CALDES como en PRADES, se registró la biomasa de ramas podadas anualmente, el número de nudos resultantes una vez aplicada la poda (N. ${ }^{\circ}$ Nudos) y su diámetro. En CALDES este estudio se pormenorizó a tramos de $50 \mathrm{~cm}$.

El ensayo de ONTINAR se evaluó una sola vez, a los 10 años de edad, registrándose el diámetro en cruz a $1.30 \mathrm{~m}$ y la altura total y de fuste. Los diámetros de copa se obtuvieron mediante el análisis de fotografías referenciadas (software Image J). La caracterización de la nudosidad del fuste se realizó mediante un conteo de cicatrices de poda, tanto cerradas como abiertas, en tramos de $50 \mathrm{~cm}$ hasta los 3 metros de altura.

La producción de avellanas se estimó a partir de series históricas de este cultivo, construidas en relación a la edad, expansión de la mata y la densidad de plantación (Tous et al., 2001; Tous y Rovira, 2004).

\subsection{Análisis estadístico}

Los caracteres estudiados considerados como cuantitativos se trataron mediante un análisis de varianza (Mixed model, JMP ${ }^{\circledR}$ 8.0.1), siguiendo el diseño en bloques establecido, e independientemente para cada ensayo. En el caso de ONTINAR se compararon las series de datos para cada carácter y tratamiento. Todas las diferencias significativas se consideraron para $\alpha=0.05$.

\section{Resultados}

\subsection{Crecimiento}

Los crecimientos se resumen en la Tab. 3 mostrándose los datos a los 8, 9 y 10 años, respectivamente en CALDES, PRADES y ONTINAR. El diámetro normal medio de los nogales en CALDES y PRADES fue de unos $8 \mathrm{~cm}$ lo que supone un 
crecimiento anual de alrededor de $1 \mathrm{~cm}$, mientras que en la plantación de ONTINAR el diámetro normal medio superó $\operatorname{los} 21 \mathrm{~cm}$, lo que corresponde a un incremento de $2.1 \mathrm{~cm}$ año ${ }^{-1}$. Las alturas fueron también más del doble en la plantación de ONTINAR $(12.7 \mathrm{~m})$ que en los demás ensayos (5.8 m en CALDES y 4.3 en PRADES). Los avellanos siguieron una tónica similar, con un mayor crecimiento en ONTINAR, pero la respuesta entre los diferentes ensayos fue más uniforme, con incrementos medios en altura de entre $25-33 \mathrm{~cm}_{\text {año }}{ }^{-1}$.

Tabla 3. Datos dendrométricos (Media \pm Desv Std) del nogal y del avellano en cada ensayo al final del periodo de evaluación, a los 8,9 y 10 años respectivamente.

\begin{tabular}{|c|c|c|c|c|c|c|c|}
\hline & \multirow[b]{2}{*}{ Variable } & \multicolumn{2}{|c|}{ Caldes de Montbui } & \multicolumn{2}{|c|}{ Prades } & \multicolumn{2}{|c|}{ Ontinar de Salz } \\
\hline & & $\mathrm{CON}$ & SIN & $\mathrm{CON}$ & SIN & $\mathrm{CON}$ & SIN \\
\hline \multirow{4}{*}{ Nogal } & $\begin{array}{c}\text { Diámetro } \\
\text { normal }(\mathrm{mm})\end{array}$ & $81.9 \pm 20.1$ & $88.9 \pm 18.9$ & $\begin{array}{c}* \\
78.5 \pm 21.6\end{array}$ & $\begin{array}{c}* \\
90.8 \pm 24.5\end{array}$ & $211.9 \pm 20.3$ & $212.9 \pm 18.0$ \\
\hline & Altura total (m) & $5.8 \pm 1.2$ & $5.9 \pm 1.3$ & $4.2 \pm 1.5$ & $4.4 \pm 1.4$ & $12.7 \pm 0.9$ & $12.7 \pm 0.8$ \\
\hline & $\begin{array}{c}\text { Altura } \\
\text { de fuste (m) }\end{array}$ & $2.5 \pm 0.6$ & $2.7 \pm 0.5$ & $1.7 \pm 0.2$ & $1.9 \pm 1.4$ & $4.2 \pm 0.9$ & $4.4 \pm 1.0$ \\
\hline & $\begin{array}{l}\text { Proyección } \\
\text { de copa }\left(\mathrm{m}^{2}\right)\end{array}$ & $6.3 \pm 2.4$ & $6.9 \pm 2.3$ & $\begin{array}{c}* \\
5.9 \pm 2.5\end{array}$ & $\begin{array}{c}* \\
7.3 \pm 3.0\end{array}$ & $26.6 \pm 3.9$ & $26.8 \pm 3.5$ \\
\hline \multirow{3}{*}{ Avellano } & Altura total (m) & $2.6 \pm 0.6$ & & $2.3 \pm 0.8$ & & $3.3 \pm 0.6$ & \\
\hline & $\begin{array}{l}\text { Diámetro } \\
\text { copa }(\mathrm{m})\end{array}$ & $2.1 \pm 0.3$ & & $1.7 \pm 0.5$ & & $3.4 \pm 0.4$ & \\
\hline & $\begin{array}{l}\text { Proyección } \\
\text { de copa }\left(\mathrm{m}^{2}\right)\end{array}$ & $3.2 \pm 1.1$ & & $2.6 \pm 1.2$ & & $8.9 \pm 2.3$ & \\
\hline
\end{tabular}

* = nivel de significación $\mathrm{p}<0.05$

Entre el tratamiento "nogal acompañado con avellano" (CON) y el "nogal sin acompañamiento" (SIN) no se encontraron diferencias significativas en las variables de crecimiento en CALDES ni en ONTINAR. Sin embargo, en CALDES se registró una creciente diferencia diametral superior en el tratamiento SIN a partir del $5^{\circ}$ año de edad. La misma tendencia se registró en PRADES, llegando esta superioridad del tratamiento SIN a ser significativa al final del periodo de estudio, $9^{\circ}$ año (Fig 1 y Fig. 2).

\subsection{Caracterización del fuste del nogal}

La rectitud del fuste en el ensayo de CALDES fue superior en el tratamiento CON, obteniéndose el $40 \%$ de fustes completamente rectos frente al $13 \%$ obtenido en el tratamiento SIN (Fig. 3) a los 5 años.

La elipticidad del fuste evaluada en CALDES en la sección a $2.5 \mathrm{~m}$ de altura resultó ser significativamente diferente entre tratamientos, con valores de menor elipticidad en el tratamiento CON: $\mathrm{E} \% 2.5 \mathrm{~m}$-CoN $=2.6 \pm 1.7 v s$ E $\% 2.5 \mathrm{~m}$-sIN $=5.2 \pm 3.9$ (media \pm desv.). De igual forma ocurrió en ONTINAR pero en la sección del fuste 


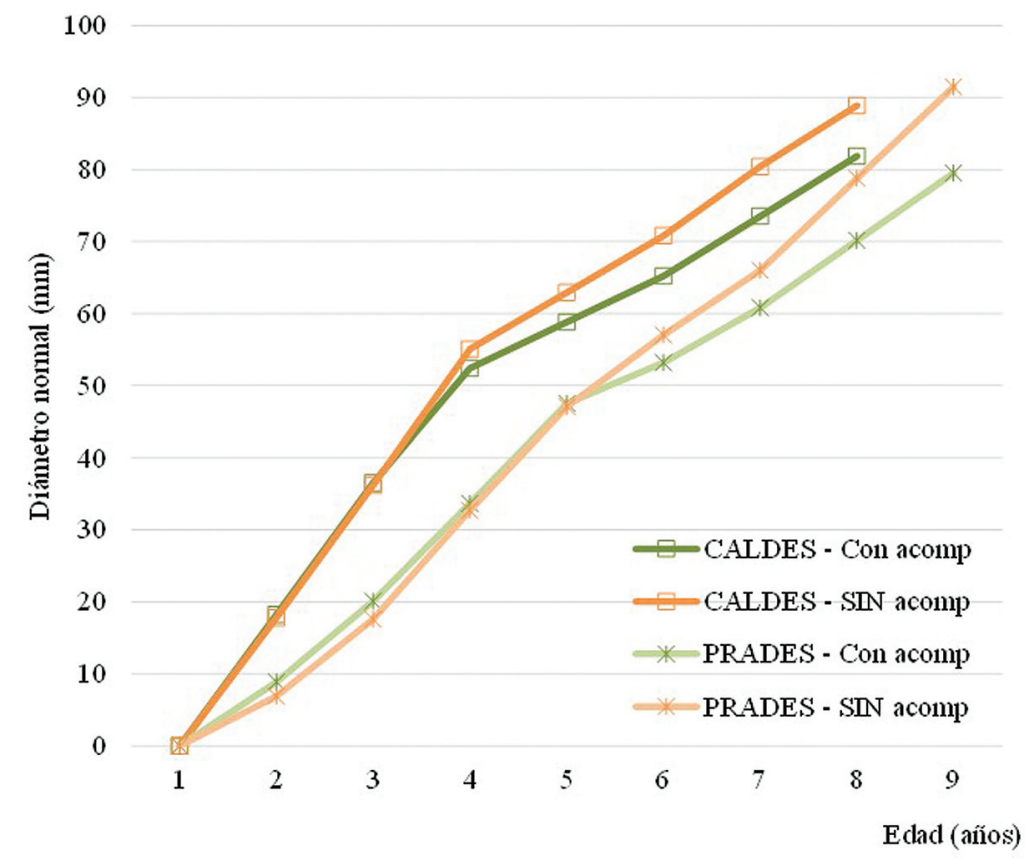

Figura 1. Evolución diametral de los nogales en CALDES y PRADES con y sin acompañamiento de avellano.

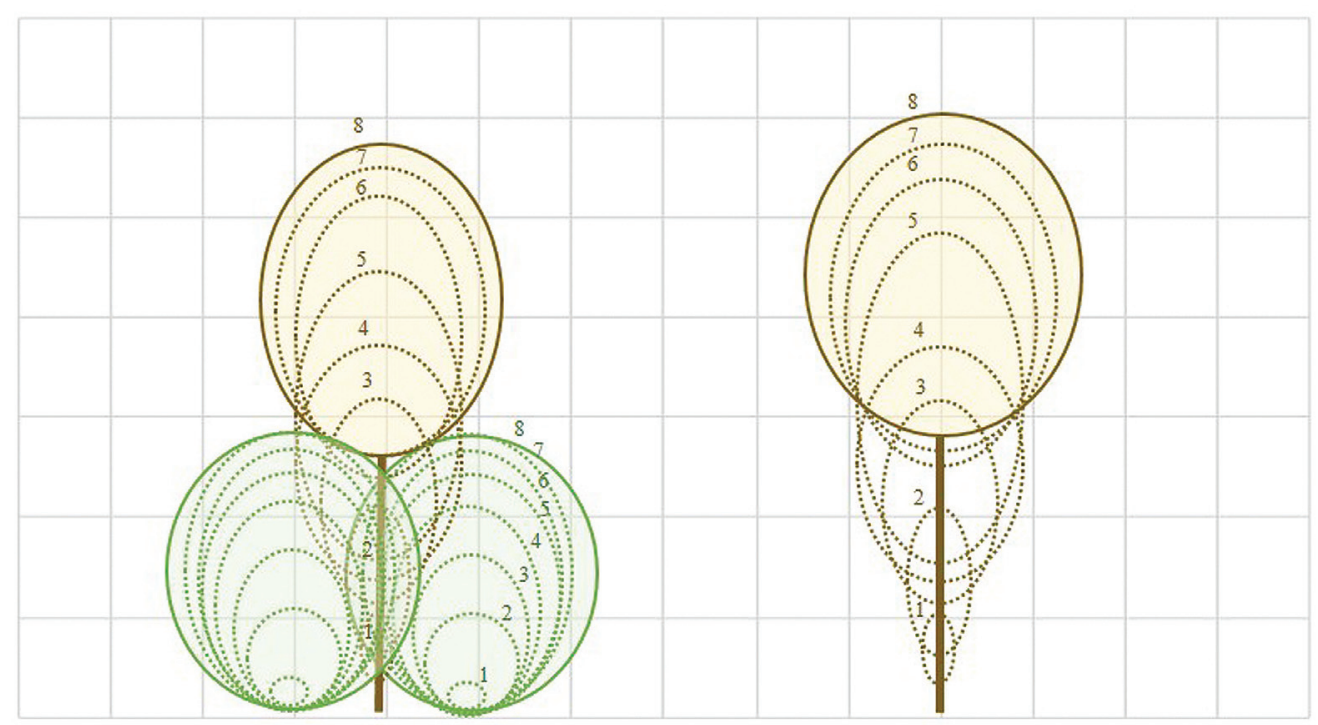

Figura 2. Gráfico de expansión de copas y fuste hasta los 8 años en CALDES en relación al tratamiento: Izquierda, nogal CON acompañamiento; Derecha, nogal SIN acompañamiento. Retícula de 1x1 m.

a $1.3 \mathrm{~m}$ de altura $(\mathrm{E} \% 1.3 \mathrm{~m}$-Con $=3.6 \pm 3.4 v s \mathrm{E} \% 1.3 \mathrm{~m}$-SIN $=4.9 \pm 4.1, \mathrm{p}<0.05)$. En este mismo punto estas diferencias no fueron significativas ni en CALDES (E\% $1.3 \mathrm{~m}$-con $=3.2 \pm 2.9 v s \mathrm{E} \% 1.3 \mathrm{~m}-\mathrm{SIN}=3.8 \pm 2.9)$ ni en PRADES $(\mathrm{E} \% 1.3 \mathrm{~m}-\mathrm{CoN}=3.6 \pm 2.9 v s \mathrm{E} \% 1.3 \mathrm{~m}-$ $\sin =4.6 \pm 2.9)$. 

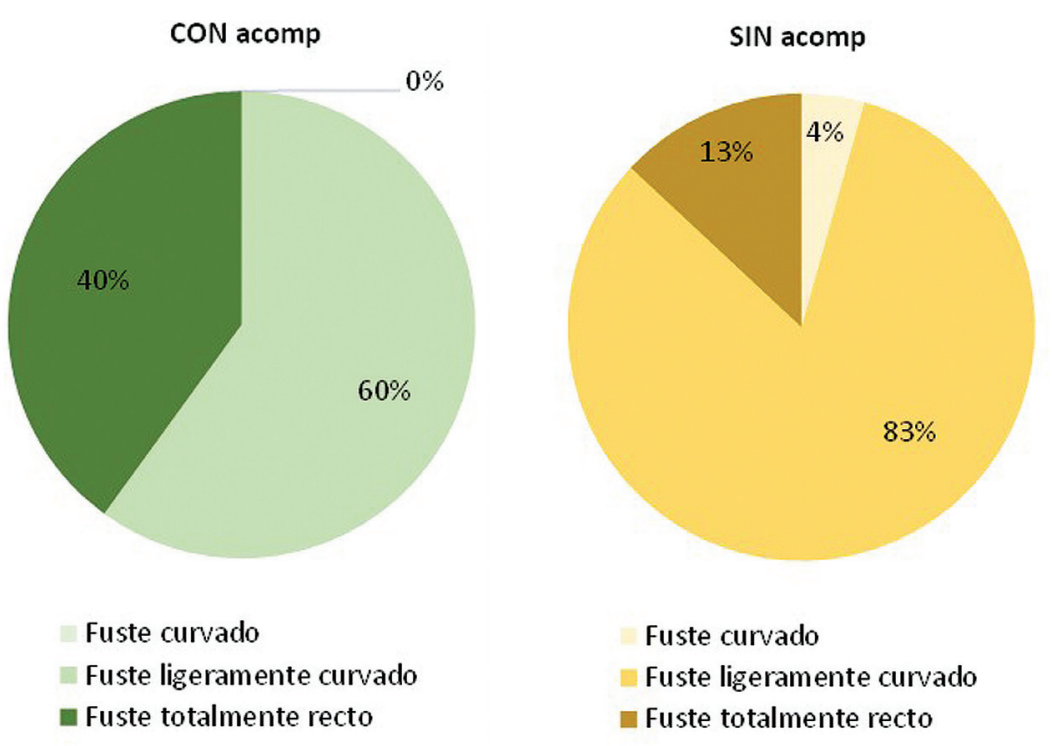

Figura 3. Porcentaje de nogales en relación a la rectitud del fuste CON y SIN acompañamiento de avellano en CALDES. Evaluación realizada a los 5 años de edad.

En PRADES el número de nudos no fue diferente entre tratamientos (N. ${ }^{\circ}$ Nudos-con $\left.=21.5 \pm 4.9 v s \mathrm{~N} .{ }^{\circ} \mathrm{Nudos}-\mathrm{sin}=23.3 \pm 6.3\right)$. En CALDES los resultados fueron semejantes $\left(\mathrm{N} .{ }^{\circ} \mathrm{Nudos}\right.$-con $\left.=23.8 \pm 8.2 v s \mathrm{~N} .{ }^{\circ} \mathrm{Nudos}-\mathrm{sin}=27.7 \pm 9.5\right)$ pero la evaluación anual a diferentes alturas permitió observar una tendencia hacia la reducción en el número de nudos en los tramos altos de los fustes CON (Fig. 4), al igual que en ONTINAR.

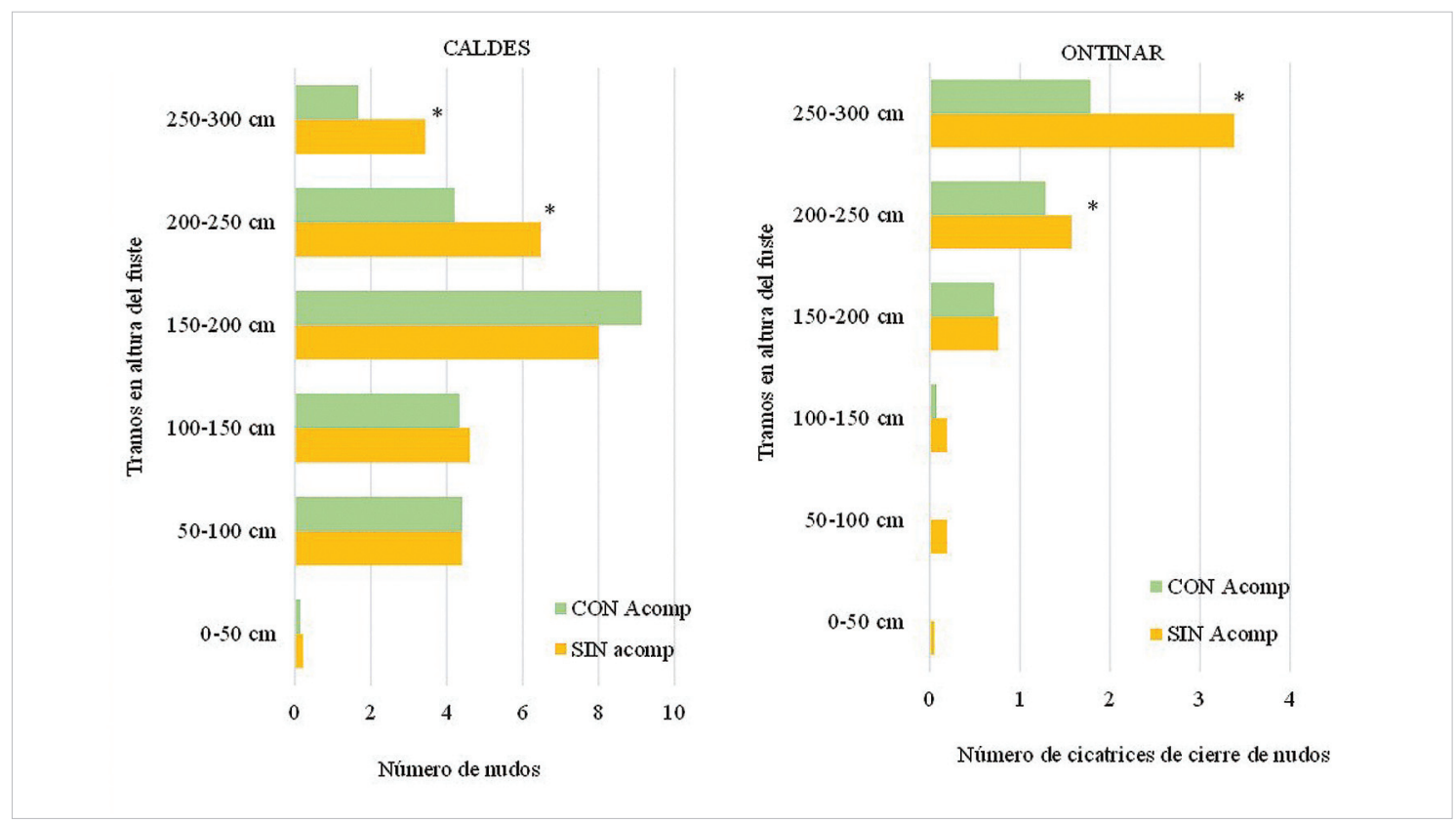

Figura 4. Número de nudos observados sobre el primer tramo de fuste de $3 \mathrm{~m}$ en CALDES hasta los 8 años (izquierda) y número de cicatrices procedentes del cierre de nudos en ONTINAR a los 10 años (derecha). 
El diámetro de los nudos en CALDES y PRADES fue un 24 y un $22 \%$ de media, respectivamente, menor con el acompañamiento de avellano (Fig. 5). En CALDES estas diferencias fueron significativas a los 5 y 7 años de edad pero a partir de los 8 años, dejaron de serlo. En PRADES fueron significativas a los 9 años de edad.

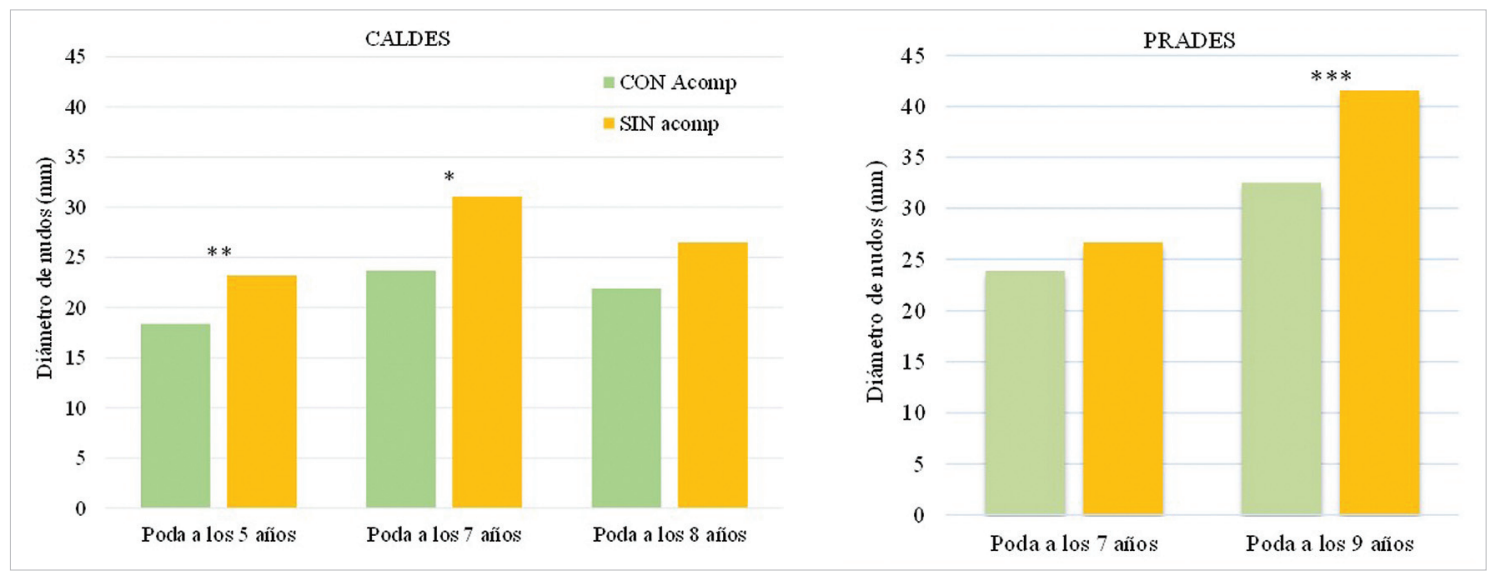

Figura 5. Diámetro de nudos sobre el primer tramo de fuste de $2.5 \mathrm{~m}$ a tres edades diferentes en CALDES y PRADES.

\subsection{Crecimiento de las variedades de avellano}

En CALDES y PRADES se observaron importantes diferencias en cuanto a vigor entre las diferentes variedades comerciales empleadas. A falta de diseño estadístico se comparó únicamente la altura de cada una de ellas (Tab. 4). Así, las variedades de mayor crecimiento fueron 'Castanyera', 'Merveille de Bowiller' y 'Segorbe' en CALDES, y 'Ennis' y 'Segorbe' en PRADES.

Tabla 4. Altura (Media \pm Desv Std) de las variedades de avellano utilizadas en los ensayos de CALDES y PRADES a los 8 y 9 años de edad respectivamente.

\begin{tabular}{|c|c|c|c|c|c|c|c|c|c|c|}
\hline Altura $(\mathrm{cm})$ & 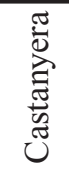 & 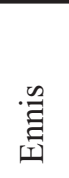 & 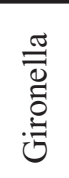 & 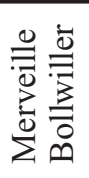 & 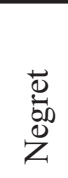 & 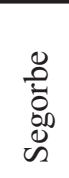 & 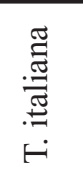 & 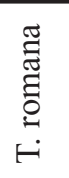 & 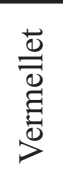 & 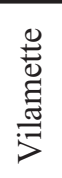 \\
\hline \multirow{3}{*}{ CALDES } & 302 & 228 & 255 & 368 & 228 & 279 & 260 & 235 & 260 & 162 \\
\hline & \pm & \pm & \pm & \pm & \pm & \pm & \pm & \pm & \pm & \pm \\
\hline & 27 & 40 & 32 & 55 & 20 & 35 & 39 & 29 & 60 & 30 \\
\hline \multirow{3}{*}{ PRADES } & 227 & 260 & & 226 & 165 & 258 & 147 & & & \\
\hline & \pm & \pm & & \pm & \pm & \pm & \pm & & & \\
\hline & 76 & 69 & & 61 & 71 & 71 & 46 & & & \\
\hline
\end{tabular}




\subsection{Biomasa podada, aporte hídrico y producción frutal}

El tratamiento CON redujo de manera significativa (36\% de media) la biomasa de ramas extraídas en la poda en CALDES y PRADES, en el periodo de 8-9 años sobre una altura de fuste de $2.5 \mathrm{~m}$ (Fig. 6).

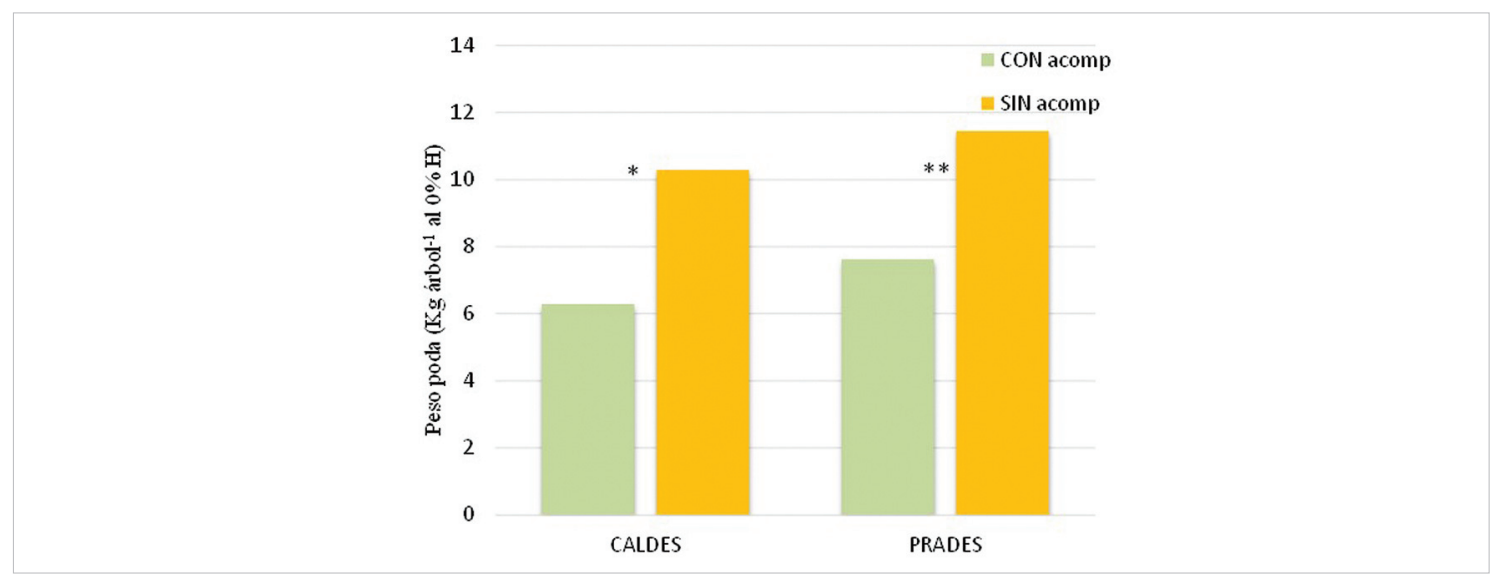

Figura 6. Peso de las ramas eliminadas sobre el primer tramo de fuste de $2.5 \mathrm{~m}$ en CALDES hasta los 8 años y en PRADES hasta los 9 años.

El riego aportado en los ensayos de CALDES y PRADES se resume en la Fig. 7. Los árboles del tratamiento SIN recibieron un 35\% menos de agua. Los aportes absolutos al noveno año de edad fueron de 1550 y $950 \mathrm{~m}^{3} \mathrm{ha}^{-1} \mathrm{año}^{-1} \mathrm{CON}$ y SIN respectivamente.

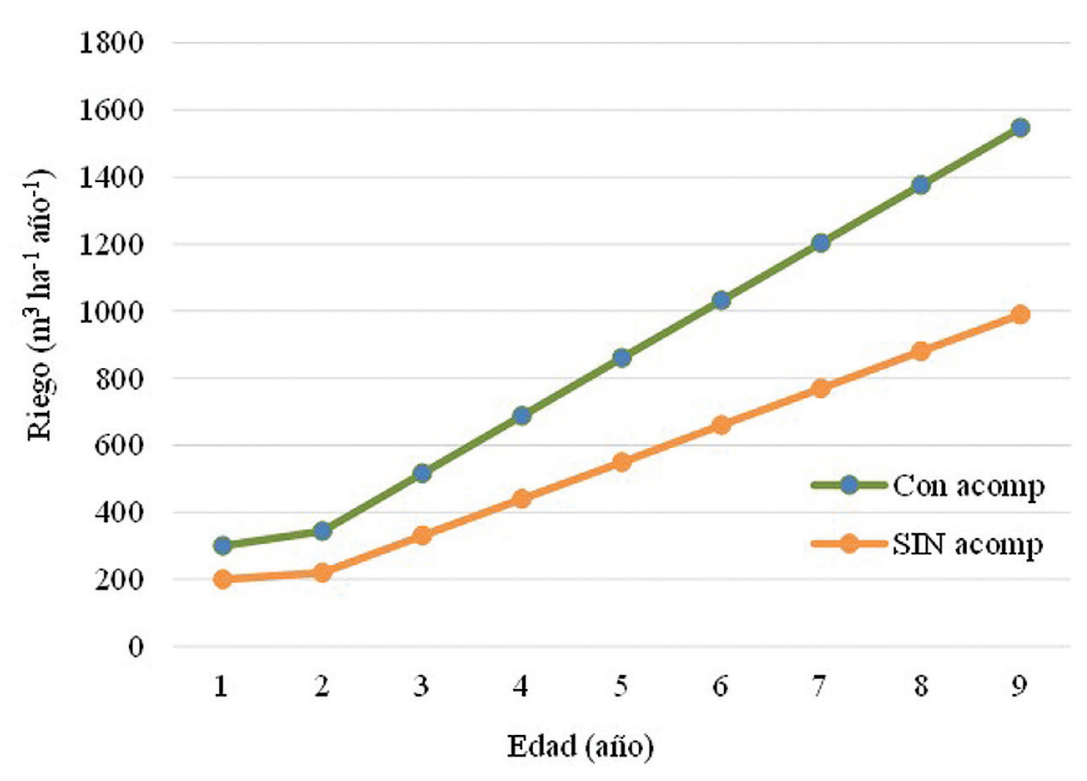

Figura 7. Volumen de riego anual medio suministrado en cada tratamiento en CALDES y PRADES. 
La producción de avellanas fue estimada en relación a la edad de los árboles y a la densidad de plantación sobre los datos de Tous et al. (2001), y de Tous y Rovira (2004) cifrada a los 10 años en unos $2000 \mathrm{~kg} \mathrm{ha}^{-1}$.

\section{Discusión}

Los crecimientos en diámetro, altura total y altura de fuste alcanzados por los nogales se sitúan en los extremos del rango (inferior en el caso de CALDES y PRADES y superior en el caso de ONTINAR) propuesto por diferentes autores (Becquey, 1997; Aletà et al., 2003; Arnold et al., 2011; Vilanova et al., 2011). No obstante, el efecto del acompañamiento fue relativamente constante en todos los ensayos.

Pese a que el acompañamiento de avellano no ha dado lugar a diferencias diametrales ni en CALDES ni en ONTINAR, la Fig. 1 muestra como en CALDES la diferencia a favor del tratamiento SIN se fue acentuando con la edad. Hasta ahora esta diferencia sólo fue significativa en las condiciones del ensayo de PRADES a los 9 años de edad. En este contexto cabría cuestionarse la idoneidad de la metodología empleada para calcular las dosis de riego aplicadas en CALDES y PRADES a estas edades, resultando muy probablemente insuficientes para sostener a su máximo las producciones del nogal y avellano. Un aumento de $560 \mathrm{~m}^{3} \mathrm{ha}^{-1}$ año ${ }^{1}$ sobre los $990 \mathrm{~m}^{3} \mathrm{ha}^{-1}$ año $^{-1}$ que se incorporaron al nogal SIN, fueron insuficientes para el consumo extra de agua requerido por las dos matas de avellano. Esta reflexión es tanto más importante cuando se pretende añadir una producción comercializable de avellanas. En la fase juvenil las dotaciones hídricas calculadas en base a la proyección de copa fueron suficientes para las dos especies, lo que se comprobó en CALDES al lograr tasas de crecimiento diametral de $2 \mathrm{~cm}$ año- 1 en el nogal en ambos tratamientos. Es a partir de la entrada en producción del avellano que debería haberse aumentado sustancialmente la dotación de agua en ambas plantaciones. En ONTINAR con una aportación de agua muy superior, los crecimientos del nogal en diámetro no se vieron afectados en ningún caso por la presencia de avellanos.

El efecto del acompañamiento sobre las características del fuste del nogal fue positivo, obteniéndose fustes más rectos, menos elípticos, con menos nudos y más pequeños, especialmente a partir de los $2 \mathrm{~m}$ de altura. Algunos autores constataron resultados similares en otras estaciones (Tani et al., 2006; Clark et al., 2008; Pelleri et al., 2013). Se constata cómo los 8-10 años es un plazo adecuado para valorar la rectitud y las características de los nudos en los 3 primeros metros de fuste puesto que la mayoría de árboles superan sobradamente esta altura. Independientemente de la evolución que pueda tener la rectitud hasta las primeras claras o hasta final de turno (Macdonald et al., 2001), triplicar la cantidad de fustes con rectitud completa en la zona de madera más valiosa (primeros $3 \mathrm{~m}$ de fuste) como se logra en el tratamiento $\mathrm{CON}$, influirá considerablemente en la tasación económica final de la plantación. La reducción del diámetro de nudos obtenida con el acompañamien- 
to, en valores medios del $20 \%$, podría favorecer una cicatrización más rápida de los cortes, minimizándose algunos defectos sobre la madera habituales después de la poda (i.e. coloración y/o podredura descendente, entrada de xilófagos o quemaduras solares).

Muchos de los citados efectos sobre el fuste pueden relacionarse con la función de "barrera o protección" prestada por el acompañamiento. A mayor vegetación en el sotobosque de la plantación, se reduce la temperatura e insolación sobre el fuste, lo que puede evitar los problemas de quemaduras solares a las que es sensible $J u$ glans regia (Becquey, 1997). Además, se atenúan las cargas de viento laterales y se estructuran las raíces del nogal en estratos más profundos, al situarse las raíces de avellano mayoritariamente en la parte superior del perfil (i.e. 45-55 cm) (Germain and Sarraquigne, 2004). De la observación en los distintos ensayos y de los resultados obtenidos (i.e. disminución de ramas sólo a partir de los $2 \mathrm{~m}$ de fuste, Fig. 4), se deduce que la barrera física positiva que ejerció el avellano sobre el nogal llegó algo más tarde de lo deseado. El crecimiento primario del nogal fue muy superior al del avellano en las fases iniciales y así la formación de los primeros metros de fuste se generó en ausencia del efecto de "protección" (tasas alrededor de $1 \mathrm{~m}$ año ${ }^{-1}$ de altura en nogal frente a $0.5 \mathrm{~m}_{\text {año }}{ }^{-1}$, en avellano). La elección de variedades de la especie acompañante vigorosas y bien adaptadas a la estación es por tanto de gran importancia. Por otra parte, se observó que una menor distancia de los avellanos al nogal, plantados a $0.75 \mathrm{~m}$ en CALDES frente a $1.50 \mathrm{~m}$ en PRADES, permitió avanzar temporalmente el "envolvimiento" del tronco del nogal por el avellano, que en CALDES se produjo unos dos años antes que en PRADES.

Este estudio habilita muchos de los beneficios observados en plantaciones mixtas o con acompañamiento (Nichols et al., 2006) y añade algunas nuevas como la disminución de los costes de poda. En nuestro caso se obtuvo un 36\% menos de biomasa eliminada en la poda gracias al acompañamiento. Sin embargo, en el balance de los ensayos se muestra que los insumos de agua fueron 50\% superiores en el complejo nogal-avellano respecto al caso monoespecífico. El riego a partir de los 4-5 años fue insuficiente y marca unos límites básicos en la disponibilidad hídrica a la hora elegir una estación o diseñar y planificar una plantación con acompañamiento de avellano. Este apunte se confirma también a partir del ensayo de ONTINAR, en el que con un riego a manta de unos $6000 \mathrm{~m}^{3} \mathrm{ha}^{-1} \mathrm{año}^{-1}$ no existieron mermas en el desarrollo del nogal con avellano respecto al no acompañado. Por tanto, ante la planificación de una plantación mixta, es conveniente descartar estaciones de pluviometría inferior a la asimilable a $3000 \mathrm{~m}^{3} \mathrm{ha}^{-1}$ año $^{-1}$ durante el periodo vegetativo si no se puede compensar mediante el riego.

De lo anterior se desprende que este tipo de plantaciones con acompañamiento son complejas de gestionar, ya que están presentes dos especies con necesidades diferentes. Si el acompañamiento no presenta un crecimiento acompasado con la especie principal el efecto positivo puede desaparecer o convertirse en negativo (ex. inducción de curvaturas debida a un acompañamiento asimétrico por falta de una mata en un lateral del fuste, además del incremento en el coste de instalación y mantenimiento). Finalmente, la introducción de una especie frutal en el sistema, repre- 
senta en muchos sitios un reclamo para cierto tipo de fauna, p.e. ungulados, que puede ocasionar pérdidas de producción y daños como rotura de ramas e instalaciones de riego. La gestión de estas plantaciones agroforestales obliga a implementarlas con manejos dirigidos a favorecer la producción agrícola: control de plagas y enfermedades, protección contra cierto tipo de fauna, adecuado manejo de los recursos hídricos y aportes orgánicos periódicos. La sostenibilidad económica del sistema pasa necesariamente por obtener un producto agrícola anual vendible en el mercado.

\section{Conclusiones}

El escenario global actual demanda un aumento de la productividad del espacio rural conservando la sostenibilidad medioambiental de los cultivos. Los sistemas agroforestales como el descrito pueden contribuir a hacer frente a estos retos aunque deben aun desarrollarse fórmulas específicas para cada combinación agrícola-forestal y estación en particular. En este trabajo se ha demostrado que la introducción desde el inicio de una especie productora de fruto como el avellano en una plantación de nogal para madera no sólo es compatible a nivel de crecimiento y producción frutal sino que además mejora aspectos cualitativos del fuste del nogal y reduce los esfuerzos de poda. Sin embargo, es un sistema que requiere un aporte hídrico superior y un incremento de la gestión para conseguir una producción frutal competitiva. Los resultados reflejan el comportamiento del sistema en un primer tercio del turno teórico del nogal, por lo que será necesario estudiar los efectos y balance de la combinación hasta el final de éste.

\section{Agradecimientos}

A los proyectos INIA RTA2005-00057-C05 y RTA2010-00104-00 en los que se establecieron los ensayos de Caldes de Montbui y Prades, al propietario de éste último, y a la empresa 'Nogales de Aragón' por facilitar la toma de datos en su plantación de Ontinar de Salz.

\section{Bibliografía}

Aletà, N., Ninot, A., Voltas, J., 2003. Caracterización del comportamiento agroforestal de doce genotipos del nogal (Juglans sp.) en dos localidades de Cataluña. Inv. Agrar. Sist. Rec. F. 12(1), 39-50.

Aletà, N., Vilanova, A., 2014. Les plantacions espanyoles d'alt valor. Catalunya Forestal 119, 21-24.

Arnold, E., Frank, R., Hein, S., Ehring, A., 2011. Croissance, qualité et mortalité du noyer hybride sur différentes stations dans le Bade-Wurtemberg (Allemagne). Rev. For. Fr. 63(4), 425-434. https://doi.org/10.4267/2042/45826

Becquey, J., 1997. Les noyers à bois. Institut pour le Développement Forestier. 
Becquey, J., 2006. Des billes de noyer plus longues avec l'acompagnement ligneux. Forêt enterprise. 170(5), 45-50.

Chifflot, V., Bertoni, G., Cabanattes, A., Gavaland, A., 2006. Beneficial effects of intercropping on the growth and nitrogen status of young wild cherry and hybrid walnut trees. Agrofor. Syst. 66(1), 13-21. https://doi.org/10.1007/s10457-005-3650-3

Clark, J.R., Hemery, G.E., Savill, P.S., 2008. Early growth and form of common walnut (Juglans regia L.) in mixture with tree and shrub nurse species in southern England. Forestry 81(5), 631-642. https://doi.org/10.1093/forestry/cpn036

Germain, E., Sarraquigne, J.P., 2004. Le noisetier. Ctifl, Paris.

Nichols, J.D., Bristow, M., Vanclay, J.K., 2006. Mixed species plantations: Prospects and challenges. For. Ecol. Manage. 233(2-3), 383-390. https://doi.org/10.1016/j.foreco. 2006.07.018

Nosenzo, A., Boetto, G., Meloni, F., 2011. La classificazione degli asortimenti ritraibili come strumento di analisi della produttività di impiantari per l'arboricoltura da legno Ed. SegdT. Dipartimento di Agronomia. Università di Torino, pp. 882-886.

Pelleri, F., Ravagni, S., Bianchetto, E., Bidini, C., 2013. Comparing growth rate in a mixed plantation (walnut, poplar and nurse trees) with different planting designs: Results from an experimental plantation in northern Italy. Ann. Silvic. Res. 37(1), 13-21. doi: 10. $12899 /$ asr- 750

Piotto, D., 2008. A meta-analysis comparing tree growth in monocultures and mixed plantations. For. Ecol. Manage. 255 (3-4), 781-786. https://doi.org/10.1016/j.foreco. 2007.09.065

Tani, A., Maltoni A., Mariotti B., Buresti Lattes E., 2006. Gli impianti da legno di Juglans regia realizzati nell'area mineraria di S. Barbara (AR). Valutazione dell'effetto di piante azotofissatrici accessorie. Forest. 3(4), 588-597. https://doi.org/10.3832/efor0407-003 0588

Tous, J., Rovira, M., Romero, A., 2001. Avellano. In: SECH (eds.), La horticultura española. Madrid pp. 275-278.

Tous, J., Rovira, M., 2004. Situación y perspectivas agronómicas del cultivo del avellano. Vida Rural 201, 41-45.

Vilanova, A., Garcia, D., Aletà, N., 2011. Evaluación del crecimiento y de la producción de madera en plantaciones españolas de nogal realizadas con progenies híbridas. Resultados en fase semiadulta. Navarra Forestal 28, 12-19. 
